\title{
Introduction to the Minitrack Personal Health Management and Technologies
}

\author{
Freimut Bodendorf \\ University of Erlangen- \\ Nuremberg, Germany \\ freimut.bodendorf@,fau.de
}

\author{
Nilmini Wickramasinghe \\ Epworth HealthCare and \\ Deakin University, Australia \\ nilmini.work@gmail.com
}

\author{
Tuan Huy Ma \\ Fraunhofer Institute IIS \\ Erlangen, Germany \\ tuan-huy.ma@scs.fraunhofer.de
}

Mobile Apps, wearables, and individualized services are dramatically influencing how patients and their families can manage their own health and wellness. Further, they have the potential to facilitate the design and development of superior healthcare delivery by clinicians and caregivers as well as assist in addressing many of the challenges currently facing healthcare delivery in all OECD countries. In addition, there is a trend to foster active patient participation in their care and thus we are witnessing a plethora of emerging technologies to assist in this regard. The data collected enable sophisticated services for self-care and smart decision making. In this minitrack, we focus on how such technologies might be utilized to address the challenges faced by healthcare management such as escalating cost pressures, a growing aging population, an increasing prevalence of chronic diseases, and a move to a preventive care focus. Integral to these solutions is a patient-centric view in order to satisfy consumer expectations and provide high quality care. This minitrack provides an outlet for all research focused on health and wellness related mobile technologies, applications, and services. Selected papers can be fast-tracked for a special issue in Health and Technology published by Springer.
The minitrack comprises two sessions. A first set of presentations focuses on research design and concepts as well as empirical studies in the field of wearable and mobile technologies to support personal health and fitness. The physician's perspective as well as the patient-centric view will be addressed. In a second set of presentations there is a stronger focus on the application perspective. Specific application areas and exemplary prototypical systems will be introduced. In addition, the fast growing area of datacentric management of personal health is taken into account, looking at practices of descriptive, predictive, and prescriptive analytics based on "quantified self" approaches. Taken together, the contributions serve to exhibit and illustrate the potentials and limits of modern technologies for personal health management and wellness.

For five years now the minitrack has been home to a persistent and growing community of academic researchers and healthcare professionals who are interested in the potentials of mobile technologies and services as well as in management principles for individual persons. As this field eveloves, future topics will include the Internet of Things and ecosystems of persons and autonomous technical systems. 